\title{
Diameter rigidity for Kähler manifolds with positive bisectional curvature
}

\author{
Ved Datar ${ }^{1} \cdot$ Harish Seshadri ${ }^{1}$ iD
}

Received: 28 August 2021 / Revised: 28 August 2021 / Accepted: 22 December 2021

(c) The Author(s), under exclusive licence to Springer-Verlag GmbH Germany, part of Springer Nature 2022

\section{Abstract}

We prove that a Kähler manifold with positive bisectional curvature and maximal diameter is isometric to complex projective space with the Fubini-Study metric.

\section{Introduction}

Let $(M, \omega)$ be a Kähler manifold. The bisectional curvature of $\omega$ along real unit tangent vectors $X, Y$ is defined to be

$$
\mathrm{BK}(X, Y)=\operatorname{Rm}(X, J X, J Y, Y),
$$

where Rm denotes the Riemann curvature tensor of the Riemannian metric associated to $\omega$. In this note we will be concerned with Kähler manifolds $(M, \omega)$ satisfying

$$
\mathrm{BK} \geq 1
$$

i.e., $\mathrm{BK}(X, Y) \geq 1$ for all real unit tangent vectors $X, Y$.

A diameter comparison theorem was established for compact Kähler $n$-manifolds satisfying (1) in [5]. The comparison space here is the complex projective space $\mathbb{C} P^{n}$

Communicated by Ngaiming Mok.

Research supported in part by the Infosys Young Investigator award.

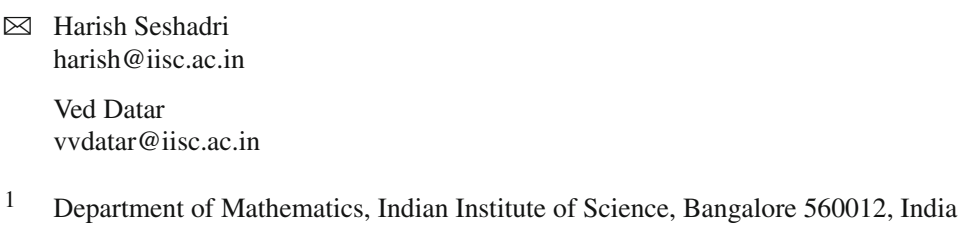


endowed with the Fubini-Study metric $\omega_{\mathbb{C} P^{n}}$, normalized so that

$$
\int_{\mathbb{C} P^{n}} \omega_{\mathbb{C} P^{n}}^{n}=(2 \pi)^{n}, \text { equivalently Ric }=(n+1) \omega_{\mathbb{C} P^{n}}
$$

Theorem 1 (Li-Wang [5]) If $\left(M^{n}, \omega\right)$ is a compact Kähler manifold satisfying $\mathrm{BK} \geq 1$, then

$$
\operatorname{diam}(M) \leq \operatorname{diam}\left(\mathbb{C} P^{n}, \omega_{\mathbb{C} P^{n}}\right)=\frac{\pi}{\sqrt{2}}
$$

Remark In [5], the diameter bound is stated to be $\pi / 2$. This is due to a different normalization for the Hermitian extension of the Riemannian metric.

The main result of this note is a characterization of the case of equality in Theorem 1:

Theorem 2 Let $\left(M^{n}, \omega\right)$ be a compact Kähler manifold satisfying $\mathrm{BK} \geq 1$. If

$$
\operatorname{diam}(M, \omega)=\operatorname{diam}\left(\mathbb{C} P^{n}, \omega_{\mathbb{C} P^{n}}\right),
$$

then $(M, \omega)$ is isometric to $\left(\mathbb{C} P^{n}, \omega \mathbb{C} P^{n}\right)$.

The diameter bound in Theorem 1 is analogous to the classical Bonnet-Myers diameter bound for compact Riemannian manifolds with positive Ricci curvature. However, one cannot relax the curvature assumption to a positive Ricci lower bound in the Kähler case, as pointed out in [6]: endow $\mathbb{C} P^{1}$ with the round metric of curvature $\frac{1}{n+1}$ and consider the product metric on the $n$-fold product

$$
M=\mathbb{C} P^{1} \times \ldots \times \mathbb{C} P^{1}
$$

The Ricci curvature of $M$ satisfies Ric $=(n+1) \omega$, but

$$
\operatorname{diam}(M)=\sqrt{\frac{n}{n+1}} \pi>\frac{\pi}{\sqrt{2}}
$$

if $n \geq 2$.

In the Riemannian case, the equality case of the Bonnet-Myers diameter bound is the well-known maximal diameter theorem of Cheng. Theorem 2 can be regarded as the Kähler analogue of Cheng's theorem.

Theorem 2 has been established under additional assumptions in [6,11]. In [6], the authors construct a totally geodesic $\mathbb{C} P^{1}$ with sectional curvature 2 and use this to show that rigidity holds if $\int_{M} \omega^{n}>\pi^{n}$. In [11], the authors assume that there are compact connected complex submanifolds $P$ and $Q$ in $M$ with $\operatorname{dim}(P)+\operatorname{dim}(Q)=n-1$ and $d(P, Q)=\frac{\pi}{\sqrt{2}}$. An eigenvalue comparison theorem is then employed to show rigidity. 
Our strategy for proving Theorem 2 is to establish a monotonicity formula for a function arising from Lelong numbers of positive currents on $\mathbb{C} P^{n}$. In [7], the $\partial \bar{\partial}$ comparison theorem of [11] is reformulated as asserting the positivity of a certain $(1,1)$-current and this is the current we work with.

\section{Lelong numbers and a monotonicity formula on $\mathbb{C} P^{n}$}

Let $M$ be a Kähler manifold. In what follows, we frequently use the real operator

$$
d^{c}=\frac{\sqrt{-1}}{2 \pi}(\bar{\partial}-\partial) .
$$

Note that

$$
d d^{c}=\frac{1}{\pi} \sqrt{-1} \partial \bar{\partial}
$$

If $T$ is a non-negative current on a $M$ such that

$$
T=d d^{c} \varphi
$$

in a neighbourhood of a point $q \in M$, then the Lelong number of $T$ at $q$ is defined as

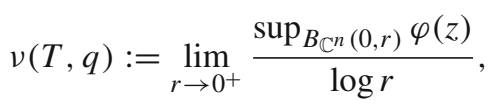

where $z$ is a holomorphic coordinate in a neighbourhood of $q$ such that $z(q)=0$. It is not difficult to see (for instance using the maximum principle) that the quotient on the right is increasing in $r$, and hence the limit $v(T, q)$ exists and is moreover non-negative and independent of the choice of holomorphic coordinates. Note that the normalization is chosen so that if $V$ is a smooth hypersurface with defining function $f$, and $[V]$ denotes the current of integration along $V$, then by the Poincáre-Lelong equation, $[V]=d d^{c} \log |f|$, and so $v([V], q)=1$ for any point $q \in V$.

The following proposition is well known (cf. [2, pg. 164-165]), but since the proof of our main theorem has a precise dependence on the constants involved, we provide a proof for the convenience of the reader.

Proposition 3 Suppose $T=d d^{c} \varphi$ as above in a neighbourhood of $q$ with holomorphic coordinates $z=\left(z^{1}, \ldots, z^{n}\right)$ such that $z(q)=0$. We then have

$$
\nu(T, q)=\lim _{r \rightarrow 0^{+}} \frac{1}{\pi^{n-1} r^{2 n-2}} \int_{B_{\mathbb{C}^{n}}(0, r)} T \wedge \omega_{\mathbb{C}^{n}}^{n-1},
$$

where $B_{\mathbb{C}^{n}}(0, r)$ is the ball of radius $r$ around the origin with respect to the Euclidean metric $\omega_{\mathbb{C}^{n}}=\frac{\sqrt{-1}}{2} \partial \bar{\partial}|z|^{2}$. 
Note that quantity on the right above is increasing in $r$ (cf. [4, pg. 390]), and hence the limit, in particular, exists.

Proof First suppose that $\varphi$ is smooth. We let

$$
\begin{aligned}
v\left(d d^{c} \varphi, 0, t\right) & :=\frac{1}{\pi^{n-1} t^{2 n-2}} \int_{B_{\mathbb{C}^{n}(0, t)}} d d^{c} \varphi \wedge \omega_{\mathbb{C}^{n}}^{n-1}, \\
\mu_{t}(\varphi) & :=\frac{1}{\sigma_{2 n-1}} \int_{\mathbb{S}^{2 n-1}} \varphi(t, \theta) d \sigma(\theta)
\end{aligned}
$$

where $\sigma_{2 n-1}=2 \pi^{n} /(n-1)$ ! is the volume of the unit sphere in $\mathbb{S}^{2 n-1} \subset \mathbb{C}^{n}$, and $d \sigma$ is the standard Riemannian measure on $\mathbb{S}^{2 n-1}$ Let $\mathbb{S}_{t}^{2 n-1}$ be the sphere of radius $t$ centred at the origin, $d \sigma_{t}$ the Riemannian measure on it and let $\partial \varphi / \partial v$ be the normal derivative of $\varphi$. Differentiating in $t$,

$$
\begin{aligned}
\frac{d \mu_{t}(\varphi)}{d t} & =\frac{1}{\sigma_{2 n-1}} \int_{\mathbb{S}^{2 n-1}} \frac{\partial \varphi}{\partial t}(t, \theta) d \sigma \\
& =\frac{1}{\sigma_{2 n-1} t^{2 n-1}} \int_{\mathbb{S}_{t}^{2 n-1}} \frac{\partial \varphi}{\partial \nu} d \sigma_{t} \\
& =\frac{2}{\sigma_{2 n-1} t^{2 n-1}} \int_{B_{\mathbb{C}^{n}(0, t)}} \Delta \bar{\partial} \varphi \frac{\omega_{\mathbb{C}^{n}}^{n}}{n !} \\
& =\frac{2}{\sigma_{2 n-1} t^{2 n-1}} \int_{B_{\mathbb{C}^{n}(0, t)}} \sqrt{-1} \partial \bar{\partial} \varphi \wedge \frac{\omega_{\mathbb{C}^{n}}^{n-1}}{(n-1) !} \\
& =\frac{2 \pi}{\sigma_{2 n-1}(n-1) !} \cdot \frac{1}{t^{2 n-1}} \int_{B_{\mathbb{C}^{n}(0, t)}} d d^{c} \varphi \wedge \omega_{\mathbb{C}^{n}}^{n-1} \\
& =\frac{v(T, 0, t)}{t} .
\end{aligned}
$$

Note that in the third line we have the $\bar{\partial}$-Laplacian $\Delta_{\bar{\partial}}$, and hence the factor of 2 on application of Green's formula. Integrating the above equality from $r$ to 1, we obtain the so-called Jensen-Lelong formula (cf. [2, pg. 163]):

$$
\mu_{1}(\varphi)-\mu_{r}(\varphi)=\int_{r}^{1} v\left(d d^{c} \varphi, 0, t\right) \frac{d t}{t} .
$$

By regularization, the above equality also holds for a general, possibly non-smooth, plurisubharmonic function $\varphi$. Changing variables $s=\log t$ and dividing by $\log r$ we have

$$
\frac{\mu_{r}(\varphi)}{\log r}=\frac{\mu_{1}(\varphi)}{\log r}-\frac{1}{\log r} \int_{\log r}^{0} v\left(d d^{c} \varphi, 0, e^{s}\right) d s
$$


and letting $r \rightarrow 0^{+}$we obtain

$$
\lim _{r \rightarrow 0^{+}} v(T, 0, r)=\lim _{r \rightarrow 0^{+}} \frac{\mu_{r}(\varphi)}{\log r} .
$$

Next proceeding as in [2, pg. 165], by Harnack inequality and maximum principle, we have that

$$
\lim _{r \rightarrow 0^{+}} \frac{\mu_{r}(\varphi)}{\log r}=\lim _{r \rightarrow 0^{+}} \frac{\sup _{z \in \partial B_{\mathbb{C}^{n}(0, r)} \varphi(z)}}{\log r}=\lim _{r \rightarrow 0^{+}} \frac{\sup _{z \in B_{\mathbb{C}^{n}}(0, r)} \varphi(z)}{\log r} .
$$

We require the following modification, which as far as we can tell, seems to be new.

Proposition 4 Let $T$ be a non-negative current on $\mathbb{C} P^{n}$ in a Kähler class, and $q \in$ $\mathbb{C} P^{n}$. Then

$$
\Theta(T, q, r):=\frac{1}{(2 \pi)^{n-1} \sin ^{2 n-2}(r / \sqrt{2})} \int_{B_{\mathbb{C} P}(q, r)} T \wedge \omega_{\mathbb{C} P^{n}}^{n-1}
$$

is increasing in $r$. Here $B_{\mathbb{C} P^{n}}(q, r)$ is the ball of radius $r$ with respect to $\omega_{\mathbb{C}} P^{n}$. Moreover, we also have that

$$
\lim _{r \rightarrow 0^{+}} \Theta(T, q, r)=v(T, q) .
$$

Note that the factor in the denominator is precisely the volume of a ball of radius $r$ in $\mathbb{C} P^{n-1}$ with respect to the Fubini-Study metric $\omega_{\mathbb{C} P^{n-1}}$ upto a factor of $(n-1)$ !.

Proof Let us first assume that $T$ is a smooth $(1,1)$ Kähler form. We use homogenous coordinates $\left[\xi_{0}: \xi_{1}: \cdots: \xi_{n}\right]$ on $\mathbb{C} P^{n}$ with $q=[1: 0: \cdots: 0]$, and the usual in-homogenous coordinates $Z_{i}=\frac{\xi_{1}}{\xi_{0}}$ on $\xi_{0} \neq 0$. Then

$$
\omega=\sqrt{-1} \partial \bar{\partial} \log |\xi|^{2}=\sqrt{-1} \partial \bar{\partial} \log \left(1+|Z|^{2}\right) .
$$

We then compute

$$
\begin{aligned}
\Theta(T, q, r)= & \frac{1}{2^{n-1} \sin ^{2 n-2}(r / \sqrt{2})} \int_{B_{\mathbb{C} P^{n}(q, r)}} T \wedge\left(d d^{c} \log |\xi|^{2}\right)^{n-1} \\
= & \frac{1}{2^{n-1} \sin ^{2 n-2}(r / \sqrt{2})} \int_{\partial B_{\mathbb{C} P^{n}(q, r)}} T \wedge d^{c} \log \left(1+|Z|^{2}\right) \\
& \wedge\left(d d^{c} \log \left(1+|Z|^{2}\right)\right)^{n-2} .
\end{aligned}
$$

Now, it is well known fact that

$$
\cos ^{2} \frac{d_{\mathbb{C} P^{n}}(q, Z)}{\sqrt{2}}=\frac{\left|\xi_{0}\right|^{2}}{|\xi|^{2}}=\frac{1}{1+|Z|^{2}}
$$


For instance exploiting the $U(n)$ symmetry one needs to check this only for $\mathbb{C} P^{1}$ which can be done easily. We then have that for any $Z \in \partial B_{\mathbb{C} P}(q, r)$,

$$
d^{c} \log \left(1+|Z|^{2}\right)=\frac{|Z|^{2}}{1+|Z|^{2}} d^{c} \log |Z|^{2}=\sin ^{2}\left(\frac{r}{\sqrt{2}}\right) d^{c} \log |Z|^{2} .
$$

Putting this back in the formula above we have that

$$
\Theta(T, q, r)=\frac{1}{2^{n-1}} \int_{\partial B_{\mathbb{C} P^{n}(q, r)}} T \wedge d^{c} \log |Z|^{2} \wedge\left(d d^{c} \log |Z|^{2}\right)^{n-2} .
$$

So if $r_{1}<r_{2}$, then integrating by parts we have

$$
\Theta\left(T, q, r_{2}\right)-\Theta\left(T, q, r_{1}\right)=\frac{1}{2^{n-1}} \int_{A_{\mathbb{C} P n}\left(q, r_{1}, r_{2}\right)} T \wedge\left(d d^{c} \log |Z|^{2}\right)^{n-1},
$$

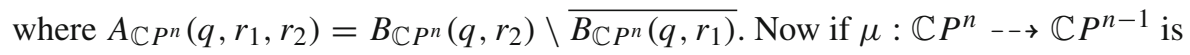
the projection from $q$ to $\left[\xi_{0}=0\right]$, then we have

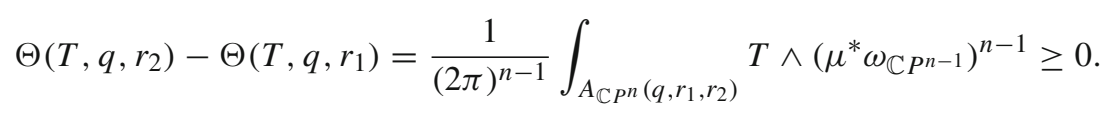

This proves the monotonicity for smooth currents. For a general positive current $T$ we can proceed by regularization. In fact in our case we can first let $r_{1}<r_{2}<R<\pi / \sqrt{2}$. Then $B(q, R)$ is contained in Euclidean ball (of radius $\tan R$ ) with respect to the inhomogenous coordinates. We can then use the standard convolution to find sequence of smooth non-negative forms $T_{j}$ converging weakly to $T$. Then since $r_{1}<r_{2}<R$,

$$
\Theta\left(T, q, r_{2}\right)-\Theta\left(T, q, r_{1}\right)=\lim _{j \rightarrow \infty}\left(\Theta\left(T_{j}, q, r_{2}\right)-\Theta\left(T_{j}, q, r_{1}\right)\right) \geq 0
$$

If $r_{2}=\pi / \sqrt{2}$, then the result follows by the monotonic convergence.

Next, to compute the limit, we again first work with smooth Kahler forms. If $T$ is smooth then in formula (3), we observe that

$$
d^{c} \log |Z|^{2}=\frac{d^{c}|Z|^{2}}{|Z|^{2}}=\frac{d^{c}|Z|^{2}}{\tan ^{2}(r / \sqrt{2})}
$$

where notice that $d(q, Z)=r$ implies that

$$
|Z|^{2}=\tan ^{2}\left(\frac{r}{\sqrt{2}}\right) .
$$

Then we have

$$
\Theta(T, q, r)=\frac{1}{2^{n-1}} \int_{\partial B_{\mathbb{C} P^{n}(q, r)}} T \wedge d^{c} \log |Z|^{2} \wedge\left(d d^{c} \log |Z|^{2}\right)^{n-2}
$$




$$
\begin{aligned}
& =\frac{1}{2^{n-1} \tan ^{2 n-2}(r / \sqrt{2})} \int_{B_{\mathbb{C} P^{n}(q, r)}} T \wedge d^{c}|Z|^{2} \wedge\left(d d^{c}|Z|^{2}\right)^{n-2} \\
& =\frac{1}{2^{n-1} \tan ^{2 n-2}(r / \sqrt{2})} \int_{B_{\mathbb{C} P^{n}(q, r)}} T \wedge\left(d d^{c}|Z|^{2}\right)^{n-1} \\
& =\frac{1}{\pi^{n-1} t^{2 n-2}} \int_{B_{\mathbb{C}^{n}(0, t)}} T \wedge \omega_{\mathbb{C}^{n}}^{n-1},
\end{aligned}
$$

where we integrated by parts in the third line and set $t=\tan (r / \sqrt{2})$, and noted that in terms of the $Z$-coordinates $B_{\mathbb{C} P^{n}}(q, r)=B_{\mathbb{C}^{n}}(0, t)$. Once again by regularization, as above, the above formula holds for general possibly non-smooth currents. Letting $t \rightarrow 0^{+}$and applying Proposition 3 we obtain (2).

Example 5 (The "model" case) On $\mathbb{C} P^{n}$ consider the current $T=\sqrt{-1} \partial \bar{\partial} \log \left|\xi_{n}\right|^{2}=$ $2 \pi\left[\xi_{n}=0\right]$, and $q=[1: 0: \cdots: 0]$. We regard this as the model case for reasons given in Section 3. Then for any $r>0$,

$$
\begin{aligned}
\int_{B_{\mathbb{C} P^{n}}(q, r)} T \wedge \omega_{\mathbb{C} P^{n}}^{n-1} & =2 \pi \int_{B_{\mathbb{C} P^{n}}(q, r) \cap\left\{\xi_{n}=0\right\}} \omega_{\mathbb{C} P^{n}}^{n-1} \\
& =2 \pi \int_{B_{\mathbb{C} P^{n-1}(q, r)}} \omega_{\mathbb{C} P^{n-1}}^{n-1} \\
& =(2 \pi)^{n} \sin ^{2 n-2}\left(\frac{r}{\sqrt{2}}\right),
\end{aligned}
$$

and so $\Theta(T, q, r)=2 \pi$ and is independent of $r$. Note that if we consider a modified

$$
\tilde{\Theta}(T, q, r):=\frac{1}{(2 \pi)^{n-1} r^{2 n-2}} \int_{B_{\mathbb{C} P^{n}(q, r)}} T \wedge \omega_{\mathbb{C} P^{n}}^{n-1},
$$

where we have $r^{2 n-2}$ in the denominator as in the usual Euclidean case, then for $T$ and $q$ as above, we would have that

$$
\tilde{\Theta}(T, q, r)=2 \pi \frac{\sin ^{2 n-2}(r / \sqrt{2})}{r^{2 n-2}} .
$$

It is easy to see that this function is decreasing in $r$. The increasing property of $\Theta(T, q, r)$ is crucial for our proof of Theorem 2 .

\section{Proof of the Theorem}

In [7], Lott introduces the following current:

$$
T_{\omega, p}:=\omega+\sqrt{-1} \partial \bar{\partial} \psi_{p}, \psi_{p}:=\log \cos ^{2}\left(\frac{d_{p}}{\sqrt{2}}\right)
$$


where $p$ is some fixed point in $M$ and $d_{p}$ is the distance function from $p$. Note that a priori, $T_{\omega, p}$ is only defined (and also smooth) away from the cut-locus of $p$. Using the Hessian comparison theorem in [11], which holds away from the cut-locus, Lott observed that $T$ is in fact a global non-negative current if $\omega$ satisfies (1).

If $\omega=\omega_{\mathbb{C} P^{n}}$, and $p=[0: 0: \cdots: 1]$, then as observed before

$$
\cos ^{2}\left(\frac{d_{\omega_{\mathbb{C} P}, p}}{\sqrt{2}}\right)=\frac{\left|\xi_{n}\right|^{2}}{|\xi|^{2}}
$$

and so

$$
T_{\omega_{\mathbb{C} P^{n}, p}}=\sqrt{-1} \partial \bar{\partial} \log \left|\xi_{n}\right|^{2}
$$

is precisely the current considered in Example 5 above.

Proof of Theorem First note that by the proof of the Frankel conjecture (cf. $[8,10]), M$ is biholomorphic to $\mathbb{C} P^{n}$. So from now on we set $M=\mathbb{C} P^{n}$. Let $p, q \in \mathbb{C} P^{n}$ such that $d_{\omega, p}(q)=\pi / \sqrt{2}$.

We claim that

$$
v\left(T_{\omega, p}, q\right)=v\left(\omega+\pi d d^{c} \psi_{\omega, p}\right) \geq 2 \pi
$$

To see this, we fix holomorphic coordinates $z:=\left(z^{1}, \ldots, z^{n}\right)$ near $q$ with $z(q)=0$. Then $C^{-1}|z(x)| \leq d(q, x) \leq C|z(x)|$ for some constant $C>0$, and hence it is enough to show that

$$
\lim _{\varepsilon \rightarrow 0^{+}} \frac{\sup _{B(q, \varepsilon)} \psi_{\omega, p}}{\log \varepsilon} \geq 2
$$

since $\omega$ being smooth does not contribute to the Lelong number. It is more convenient to work with

$$
\delta_{p}=\frac{\pi}{2}-\frac{d_{p}}{\sqrt{2}} .
$$

Then $\psi_{p}=2 \log \sin \delta_{p}$. Note that by the diameter upper bound we have $\delta_{p}(z) \geq 0$ for all $z$, and that $\delta_{p}$ is Lipshitz with constant $1 / \sqrt{2}$. Then for any $x \in \mathbb{C} P^{n}$,

$$
\delta_{p}(x)=\leq \frac{1}{\sqrt{2}} d(q, x),
$$

and so $\sup _{B(q, \varepsilon)} \psi_{\omega, p} \leq C+2 \log \varepsilon$. But then

$$
\frac{\sup _{B(q, \varepsilon)} \psi_{\omega, p}}{\log \varepsilon} \geq \frac{C}{\log \varepsilon}+2 \stackrel{\varepsilon \rightarrow 0^{+}}{\longrightarrow} 2 .
$$

But then by monotonicity, if $\omega \in c\left[\omega_{\mathbb{C} P^{n}}\right]$, putting $R=\pi / \sqrt{2}$, we have 


$$
\begin{aligned}
& 2 \pi c=\frac{1}{(2 \pi)^{n-1}} \int_{\mathbb{C} P^{n}} T \wedge \omega_{\mathbb{C} P^{n}}^{n-1}=\Theta\left(T_{\omega, p}, q, R\right) \geq \lim _{r \rightarrow 0^{+}} \Theta\left(T_{\omega, p}, q, r\right) \\
& \quad=v\left(T_{\omega, p}, q\right) \geq 2 \pi,
\end{aligned}
$$

and so $c \geq 1$. On the other hand note that the bisectional curvature lower bound gives

$$
\operatorname{Ric}(\omega) \geq(n+1) \omega,
$$

and so $c \leq 1$ since $[\operatorname{Ric}(\omega)]=(n+1)\left[\omega_{\mathbb{C} P^{n}}\right]$, and hence $c=1$. But then the lower bound on the Ricci curvature, and the $\sqrt{-1} \partial \bar{\partial}$-lemma imply that $\omega$ must be Kähler-Einstein and hence isometric to $\omega \mathbb{C} P^{n}$.

Acknowledgements We would like to thank Vamsi Pingali for his interest in the work and helpful discussions. We would also like to thank John Lott for useful comments on the first draft of the paper.

\section{References}

1. Colding, T.H.: Shape of manifolds with positive Ricci curvature. Invent. Math. 124(1-3), 175-191 (1996)

2. Demailly, J.P.: Complex analytic and differential geometry. https://www-fourier.ujf-grenoble.fr/ $\sim$ demailly/manuscripts/agbook.pdf

3. Demailly, J.P.: Pseudoconvex-Concave Duality and Regularization of Currents. Several Complex Variables (Berkeley, CA, 1995-1996), 233-271, Math. Sci. Res. Inst. Publ., 37, Cambridge Univ. Press, Cambridge (1999)

4. Griffiths, P., Harris, J.: Principles of Algebraic Geometry, Reprint of the 1978 Original. Wiley Classics Library, Wiley, New York (1994).. (xiv+813 pp. ISBN: 0-471-05059-8)

5. Li, P., Wang, J.: Comparison theorem for Kähler manifolds and positivity of spectrum. J. Differ. Geom. 69(1), 43-74 (2005)

6. Liu, G., Yuan, Y.: Diameter rigidity for Kähler manifolds with positive bisectional curvature. Math. Z. 290(3-4), 1055-1061 (2018)

7. Lott, J.: Comparison geometry of holomorphic bisectional curvature for Kähler manifolds and limit spaces, to appear in Duke Math J. arXiv:2005.02906

8. Mori, S.P.: Projective manifolds with ample tangent bundles. Ann. Math. (2) 110(3), 593-606 (1979)

9. Ni, L.: A monotonicity formula on complete Kähler manifolds with nonnegative bisectional curvature. J. Am. Math. Soc. 17(4), 909-946 (2004)

10. Siu, Y.T., Yau, S.T.: Compact Kähler manifolds of positive bisectional curvature. Invent. Math. 59(2), 189-204 (1980)

11. Tam, L.-F., Yu, C.: Some comparison theorems for Kähler manifolds. Manuscripta Math. 137(3-4), 483-495 (2012)

12. Zhang, K.: On the optimal volume upper bound for Kähler manifolds with positive Ricci curvature, (with an appendix by Liu, Y.). arXiv:2001.04169v2

Publisher's Note Springer Nature remains neutral with regard to jurisdictional claims in published maps and institutional affiliations. 